\title{
Engineering metabolic systems for production of advanced fuels
}

\author{
Yajun Yan · James C. Liao
}

Received: 7 December 2008 / Accepted: 14 January 2009 / Published online: 7 February 2009

(C) The Author(s) 2009. This article is published with open access at Springerlink.com

\begin{abstract}
The depleting petroleum storage and increasing environmental deterioration are threatening the sustainable development of human societies. As such, biofuels and chemical feedstocks generated from renewable sources are becoming increasingly important. Although previous efforts led to great success in bio-ethanol production, higher alcohols, fatty acid derivatives including biodiesels, alkanes, and alkenes offer additional advantages because of their compatibility with existing infrastructure. In addition, some of these compounds are useful chemical feedstocks. Since native organisms do not naturally produce these compounds in high quantities, metabolic engineering becomes essential in constructing producing organisms. In this article, we briefly review the four major metabolic systems, the coenzyme-A mediated pathways, the keto acid pathways, the fatty acid pathway, and the isoprenoid pathways, that allow production of these fuel-grade chemicals.
\end{abstract}

\section{Introduction}

The depleting petroleum reserve, recurring energy crisis, and global climate change are reigniting the enthusiasm for seeking sustainable technologies for replacing petroleum as a source of fuel and chemicals. In the past few decades, efforts in the development of bio-ethanol as an alternative fuel have led to significant success [14-16, 19]. In 2007, 6.5 billion gallons of bio-ethanol was produced in the United State [5]. However, bio-ethanol exhibits some

Y. Yan · J. C. Liao $(\bowtie)$

Department of Chemical and Biomolecular Engineering, University of California at Los Angeles, 5531 Boelter Hall, 420 Westwood Plaza, Los Angeles, CA 90095, USA

e-mail: Liaoj@ucla.edu limitations, such as low energy density, high vapor pressure, and corrosiveness, which prevent its widespread utilization given the existing infrastructure.

Higher alcohols (with more than two carbons), biodiesels, and fatty acid derivatives are thought to be more suitable fuels. Their physicochemical properties are more compatible with gasoline-based fuels and allow direct utilization of existing infrastructure for storage and distribution. Furthermore, some of these fuel molecules also serve as important chemical feedstocks. Although the individual biochemical steps for synthesizing these compounds in microbes have been described previously, efforts in putting together highly productive metabolic systems have only begun recently. In this article, we first summarize the metabolic networks for producing these compounds and then review efforts in engineering the non-native producing organism, Escherichia coli. The metabolic networks discussed include the traditional butanol pathway in Clostridium species, the keto acid pathways for higher alcohols, the isoprenoid pathways, and the fatty acid biosynthesis.

\section{The coenzyme-A-dependent fermentative pathways}

Among the higher alcohols, $n$-butanol and isopropanol are the only two that are overproduced in nature by Clostridium species. $n$-Butanol has been produced by Clostridium in acetone-butanol-ethanol (ABE) fermentation. The fermentative pathway (Fig. 1) in this organism starts from acetyl-CoA. The enzyme acetyl-CoA acetyltransferase, also known as thiolase, condenses two molecules of acetyl-CoA to one molecule of acetoacetyl-CoA. From this molecule, the pathway branches into isopropanol and $n$-butanol. For the isopropanol biosynthesis, an acetoacetyl-CoA transferase (ACoAT) transfers the CoA group 


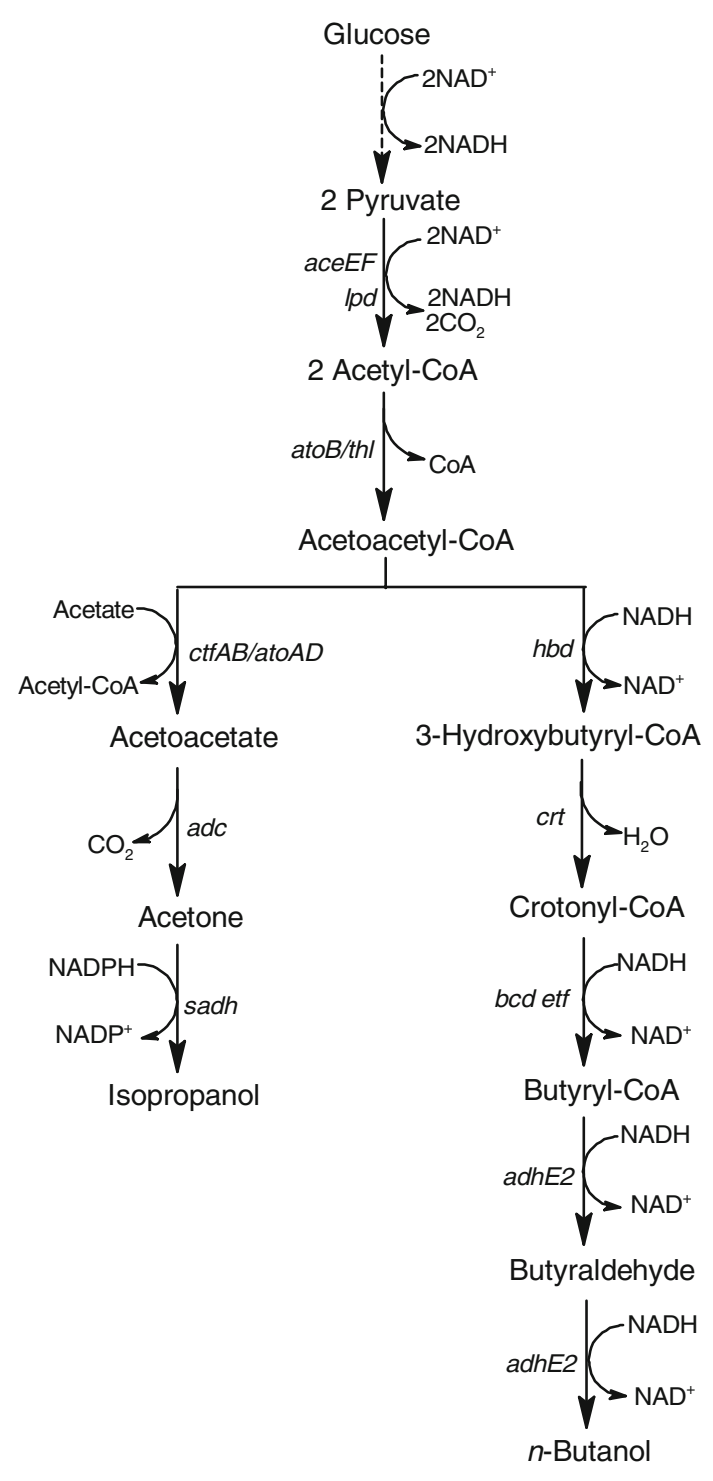

Fig. 1 Metabolic pathways for isopropanol and 1-butanol production in engineered E. coli. The dashed line indicates omitted steps. Isopropanol pathway consists of four enzymatic steps from acetylCoA. 1-Butanol pathway consists of six enzymatic steps. aceEF and lpd encode pyruvate dehydrogenase; atoB/thl encodes acetyl-CoA acetyltransferase; ctfAB/atoAD encodes acetoacetyl-CoA transferase; $a d c$, acetoacetate decarboxylase; sadh encodes secondary alcohol dehydrogenase; hbd encodes 3-hydroxybutyryl-CoA dehydrogenase; crt encodes crotonase; $b c d$ encodes butyryl-CoA dehydrogenase; etf encodes electron transfer flavoprotein; adhE2 encodes aldehyde/ alcohol dehydrogenase

away from acetoacetyl-CoA to acetate or butyrate, forming acetoacetate. The acetyl-CoA is recycled back to acetate by the combined phosphotransacetylase and acetate kinase reaction. Further, acetoacetate is decarboxylated to acetone by an acetoacetate decarboxylase (ADC). Then acetone is reduced to isopropanol by a NADPH-dependent secondary alcohol dehydrogenase (SADH) [12].

For $n$-butanol biosynthesis, acetoacetate has to go through four steps of NADH-dependent reduction and one step of dehydration. Acetoacetate is first reduced to 3-hydroxybutyryl-CoA by 3-hydroxybutyryl-CoA dehydrogenase (HBD). Then, 3-hydroxybutyryl-CoA is dehydrated to crotonyl-CoA by a crotonase (CRT). Third, a butyrylCoA dehydrogenase (BCD) catalyzes the reduction of crotonyl-CoA to butyryl-CoA. Finally, an aldehyde/alcohol dehydrogenase (AADH) converts butyryl-CoA to $n$-butanol through two consecutive reduction reactions.

\section{Isopropanol production in Escherichia coli}

The secondary alcohol, isopropanol, is both a desirable fuel and an important chemical feedstock in the petrochemical industry. Its dehydrated product, propylene, serves as the monomer for making polypropylene. In addition, it can be used as an additive to petroleum-based fuels. Replacing methanol with isopropanol in the esterification process of fat and oil could generate crystallization-resistant biodiesels [12].

As described above, isopropanol is produced by Clostridium species in nature. However, as a native metabolite, it can only be produced in a limited amount for the hosts' own benefits as a detoxification response to low $\mathrm{pH}$ conditions. The maximum titer reported in its native producer, Clostridium, was $1.8 \mathrm{~g} / \mathrm{l}$ [9]. To improve the production of isopropanol, the fully characterized isopropanol biosynthetic pathway (Fig. 1) was reconstructed in the genetic tractable host E. coli [12].

Escherichia coli has been reported to produce acetone [6], the immediate precursor of isopropanol, by expressing the intact pathway from Clostridium acetobutylicum ATCC824 consisting of the acetyl-CoA acyltransferase, ACoAT, ADC encoded by the thl, ctfAB, and $a d c$ genes, respectively. The reported titer was around $5.4 \mathrm{~g} / \mathrm{l}$, similar to the yield of native host for acetone. Furthermore, with a SADH co-expressed with the acetone pathway in E. coli, the isopropanol production was achieved [12]. The pathway efficiency was tuned by using genes from different organisms, a bio-prospecting approach. Since the genes from Clostridium usually have a low GC content, which may lead to poor expression, the $E$. coli native genes $a t o B$ and atoAD, encoding acetyl-CoA acyltransferase and ACoAT, were also tested as pathway components. Additionally, two genes from $C$. beijerinckii NRRL B593 and Thermoanaerobacter brockii HTD4, encoding SADHs, were totally synthesized with codon optimization and installed into the pathway to test for production. With these efforts, the strain with a combination of $C$. acetobutylicum thl, E. coli atoAD, C. acetobutylicum adc, and C. beijerinckii adh achieved the highest titer $(\sim 5.0 \mathrm{~g} / \mathrm{l})$. The result is promising, since it demonstrates $43.5 \%$ ( $\mathrm{mol} / \mathrm{mol})$ conversion ratio. The theoretical yield is $1 \mathrm{~mol}$ isopropanol per mole glucose.

The production of isopropanol from glucose is not redox-balanced. Four moles of NADH is produced, while 
$1 \mathrm{~mol}$ of NADPH is consumed per mole of isopropanol. Therefore, an external electron acceptor is required or a byproduct is served as an electron acceptor.

n-Butanol production in E. coli

$n$-Butanol was proposed to be one of the better substitutes for gasoline-based transportation fuel, because of its high energy density and hydrophobicity. Its energy content $(27 \mathrm{MJ} / \mathrm{l})$ is similar to that of gasoline $(32 \mathrm{MJ} / \mathrm{l})$. The high hydrophobicity enables its transportation and storage using existing petrochemical infrastructure with minimal modification. In addition, $n$-butanol has a low vapor pressure of $4 \mathrm{mmHg}$ at $20^{\circ} \mathrm{C}$, which allows its mixing with gasoline at any ratio without exceeding air quality specifications.

The microbial production of $n$-butanol has a history of over 100 years. Traditionally, $n$-butanol is produced by Clostridium species through the ABE fermentation. However, $n$-butanol production via this procedure is difficult to control and optimize, particularly because Clostridium exhibits complex physiological features, such as oxygen sensitivity, slow growth rate, and spore-forming life cycles. Thus, it is desirable to create new $n$-butanol producing organisms using metabolic engineering techniques.

Recently, $n$-butanol production in a heterologous host, E. coli, using the traditional CoA-dependent pathway originated from C. acetobutylicum (Fig. 1) was reported for the first time [2]. Atsumi et al. created two synthetic operons carrying all the essential genes ( $t h l, h b d, c r t, b c d$, etfAB, and adhE2) involved in the pathway. Co-expression of the two operons in E. coli led to the initial production of $n$-butanol at $14 \mathrm{mg} / \mathrm{l}$ anaerobically using glucose as sole carbon source. To optimize the pathway, alternative enzymes of different origins were evaluated. More specifically, with E. coli atoB gene in place of $C$. acetobutylicum thl, a more than threefold increase of $n$-butanol production was observed. However, replacing the original enzymes for conversion from crotonyl-CoA to butyryl-CoA with homologues and isoenzyme from Megasphaera elsdenii or Streptomyces coelicolor resulted in a much lower yield of $n$-butanol in E. coli. Nevertheless, this result does not exclude the possibility of the existence of other genes that might improve $n$-butanol production in $E$. coli.

Furthermore, $n$-butanol production does not simply rely on the enzyme activities. The product formation also needs sufficient carbon precursor, acetyl-CoA, and reducing power, NADH. To further improved $n$-butanol production, the host $E$. coli strain was engineered by deleting the native pathway competing for both carbon flux and reducing power. The best strain candidate, named JCL88, with the deletion of $l d h A, a d h E, f r d B C, p t a$, and $f n r$, allowed a more than twofold increase in $n$-butanol production, accompanied by the dramatic drop in the formation of lactate, acetate, ethanol, and succinate. The highest titer of $552 \mathrm{mg} / \mathrm{l}$ was reported with optimized pathway and improved strain. Although the yield was still low, this work demonstrated the feasibility of heterologous $n$-butanol production and proposed the principles for further optimization.

\section{The keto acid pathways}

Importing a non-native pathway in a heterologous host such as $E$. coli unavoidably introduces non-native metabolites and potential toxicity, in addition to difficulties in expressing heterologous enzymes. The resulting metabolic imbalance and cytotoxicity pose a barrier for large quantity production. In this context, it is desirable to seek for the pathways compatible to the host. Amino acid biosynthesis generates many keto acid intermediates. These keto acids can be converted to alcohols by introducing sequential decarboxylation and reduction catalyzed by broad-substrate-range keto acid decarboxylase (KDC) and alcohol dehydrogenase (ADH) (Fig. 2). For example, the isoleucine biosynthesis pathway generates 2-ketobutyrate and 2-keto3-methyl-valerate (KMV), which can be converted to $n$-propanol and 2-methyl-1-butanol (2MB), respectively. The valine biosynthesis pathway produces 2-ketoisovalerate (KIV), which is the precursor for isobutanol. The leucine biosynthesis pathway generates 2-keto-4-methyl-pentanoate, which is the substrate for 3-methyl-1-butanol (3MB). The phenylalanine biosynthesis pathway produces phenylpyruvate, which can lead to 2-phenylethanol. The norvaline biosynthesis pathway, which is normally a toxic sidereaction of the leucine biosynthesis, produces a substrate for $n$-butanol, 2-ketovalerate (KV) [3]. These pathways recently have been explored for production of the corresponding alcohols in $E$. coli with encouraging results.

Isobutanol production in E. coli

Isobutanol is an isomer of butanol. It has similar physicochemical properties to $n$-butanol, while having a higher octane number than $n$-butanol. Isobutanol has been identified as a minor fermentation product, but its high level production has not been reported until recently [3]. To achieve isobutanol production in a large quantity, the native $i l v I H C D$ operon from $E$. coli was first overexpressed to divert the carbon flux from pyruvate to KIV (Fig. 3), which led to isobutanol production at $1.7 \mathrm{~g} / \mathrm{l}$, about a fivefold increase over the strain without ilvIHCD overexpression. To prevent carbon leakage and reduce power waste, the previously generated knockout strain JCL88 ( $\triangle a d h E$, $\Delta l d h A, \Delta f r d A B, \Delta f n r, \Delta p t a)$ was used as host; a slight increase in isobutanol production $(2.2 \mathrm{~g} / \mathrm{l})$ was observed. Further, alsS from Bacillus subtilis was used to replace 
Fig. 2 Schematic illustration of higher chain alcohol production via keto acid pathway. $K D C$ keto acid decarboxylase, $A D H$ alcohol dehydrogenase. The two non-native steps and alcohols are highlighted in red. The amino acid pathways are indicated in blue

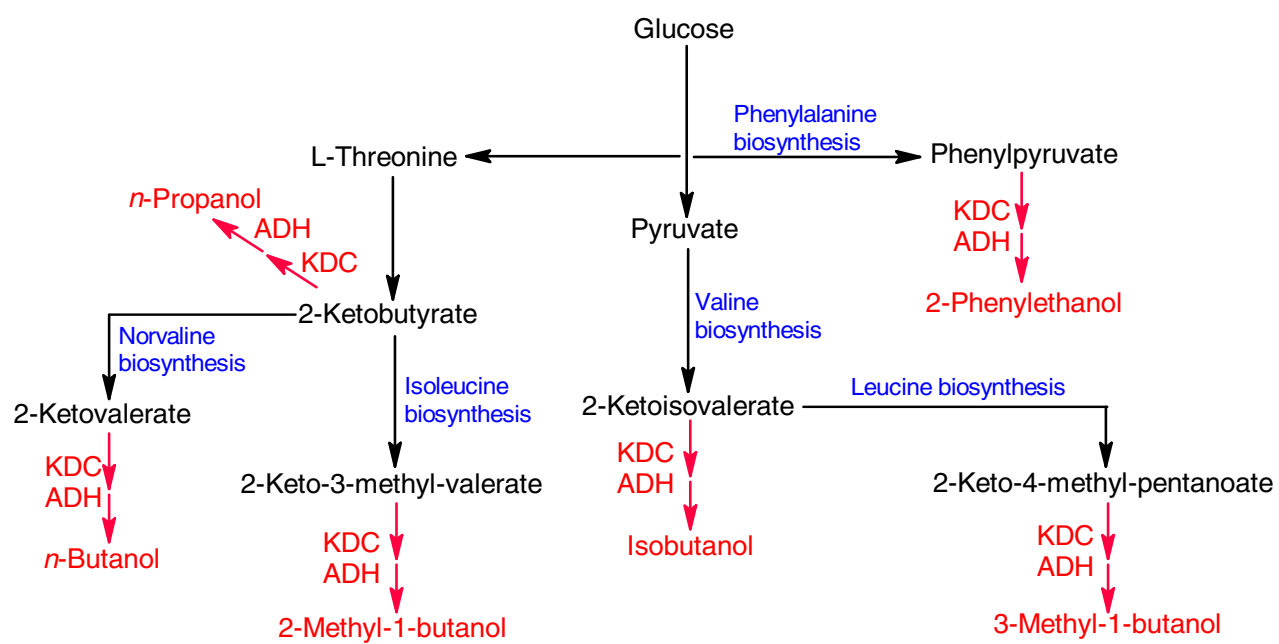

Fig. 3 Schematic illustration of the metabolic pathway from pyruvate to isobutanol and $3 \mathrm{MB}$. ilvH/als $S$ encodes acetolactate synthase; ilvC encodes acetohydroxy acid isomeroreductase; $i l v D$ encodes dihydroxy acid dehydratase; $i l v E$ encodes leucine transaminase; $\operatorname{tyr} B$ encodes leucine aminotransferase; leuA encodes 2-isopropylmalate synthase; leuCD encodes 2-isopropylmalate isomerase; leuB encodes 3-isopropylmalate dehydrogenase; kivd encodes ketoisovalerate decarboxylase; adh2 encodes alcohol dehydrogenase

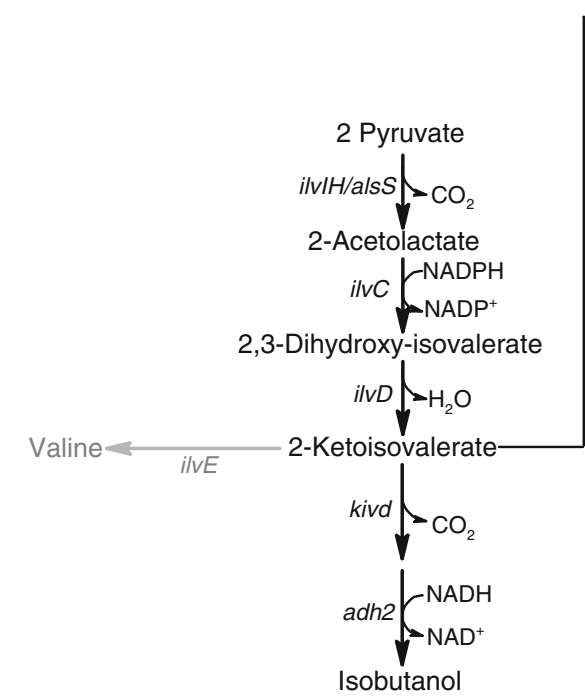

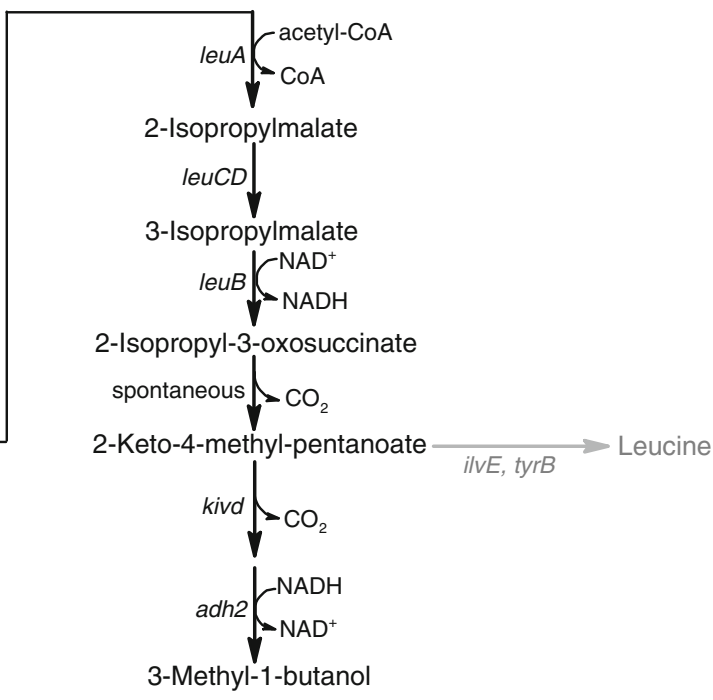

E. coli ilvIH for its high affinity towards pyruvate, which led to the isobutanol production at $3.7 \mathrm{~g} / \mathrm{l}$. In addition, $p f l B$ was deleted in strain JCL88 to conserve the pyruvate availability for KIV formation. With the combination of these overexpressions and genomic modifications, the engineered strain was able to produce isobutanol at a titer of $20 \mathrm{~g} / \mathrm{l}$ and $86 \%$ of theoretical yield (Fig. 4) [3].

Note that isobutanol is toxic to $E$. coli at a concentration $>10 \mathrm{~g} / \mathrm{l}$. However the production of isobutanol occurs mainly in the non-growing phase (Fig. 4) [3]. This result indicates that even though the cells cannot grow at the higher concentration, they nonetheless continue to produce and excrete isobutanol. Thus, even though isobutanol toxicity poses a challenge, the production level can exceed the toxicity level significantly. Mutants with higher isobutanol tolerance have been isolated [3], which also improves the productivity. Such a high-yield production demonstrates the versatility in exploring the keto acid pathways for biofuel production. The production of isobutanol $(<1 \mathrm{~g} / \mathrm{l})$ was also reported in a patent application (Donaldson et al., US patent application, US2007/0092957), which is currently under examination. Such results demonstrate that largescale commercialization of this technology is promising.

$n$-Butanol and $n$-propanol production in E. coli

As discussed above, $n$-butanol has been traditionally produced via the Clostridium pathway (Fig. 1). However, many enzymes in this pathway are oxygen sensitive and CoA-dependent, which may explain why the efficiency was low when the pathway was moved to E. coli. The keto acid pathway also paves the way for $n$-butanol production in heterologous hosts. The intermediate of norvaline biosynthesis, KV, can be channeled to $n$-butanol production by $\mathrm{KDC}$ and $\mathrm{ADH}$. Since norvaline is a toxic amino acid analogue produced only in trace amount in E. coli, both its biosynthetic pathway and the precursor need to be overexpressed. The synthesis of $\mathrm{KV}$ is from ketobutyrate through the leuABCD pathway in E. coli, where the ketobutyrate is generated from L-threonine by threonine dehydratase 

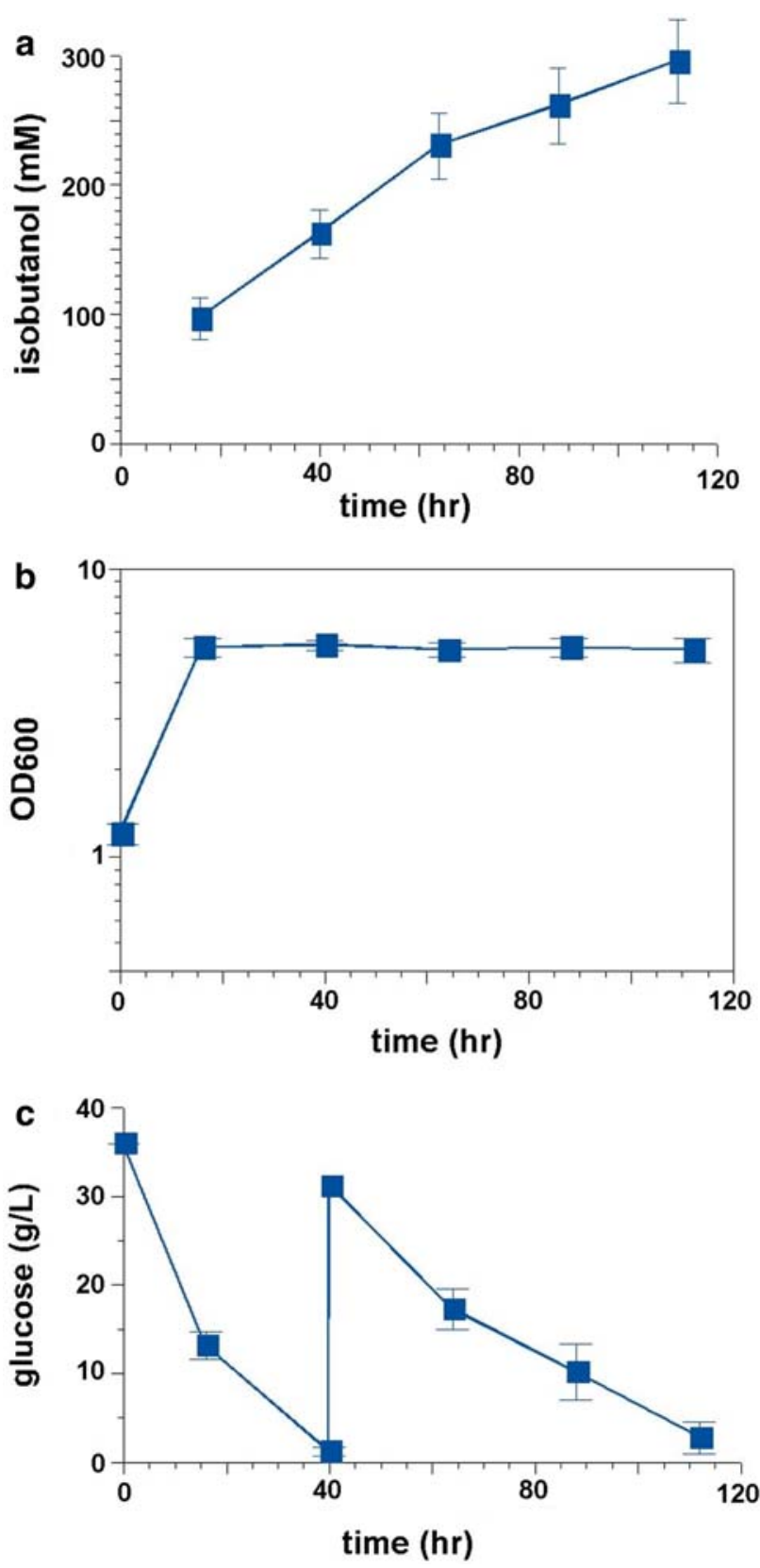

Fig. 4 Isobutanol production with engineered E. coli. Cells were grown in M9 medium containing 3.6\% glucose and $0.5 \%$ yeast extract at $30^{\circ} \mathrm{C}$; after $40 \mathrm{~h}, 3.0 \%$ glucose was added to the culture. a Isobutanol production curve. $\mathbf{b}$ Cell growth curve. $\mathbf{c}$ Time profiles of glucose concentration in medium. The figure is cited from ref. [3]

encoded by ilvA. Therefore, overexpression of these pathways is necessary. Since ketobutyrate also can be converted by $\mathrm{KDC}$ and $\mathrm{ADH}$, co-production of $n$-propanol was expected (Fig. 5).

Overexpression of ilvA-leuABCD led to a threefold increase in $n$-butanol production compared to that without overexpression. Threonine-feeding also caused a dramatic increase in $n$-butanol production, indicating the limited availability of endogenous threonine for $n$-butanol produc-

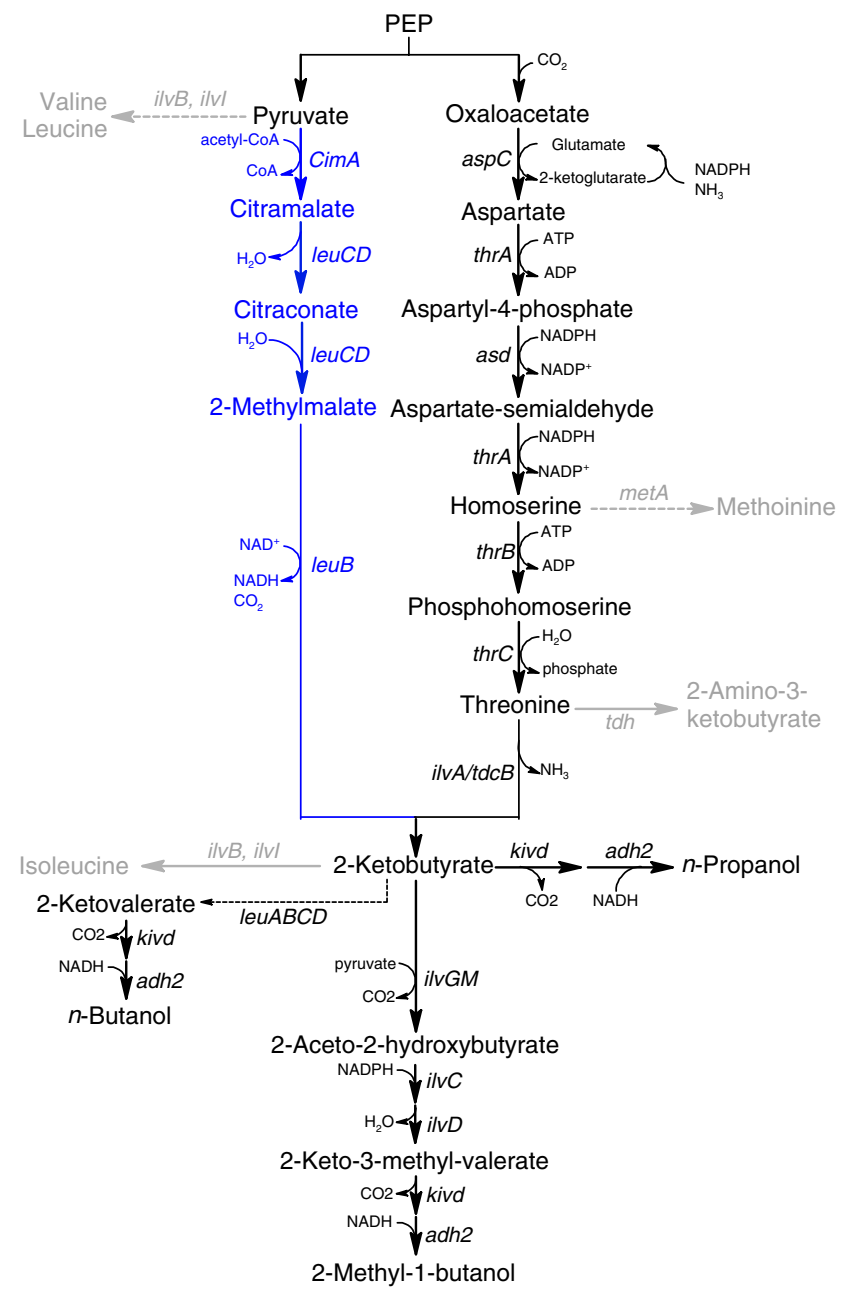

Fig. 5 Schematic illustration of ketobutyrate-mediated metabolic pathway for $n$-propanol, $n$-butanol, and $2 \mathrm{MB}$ production in $E$. coli. The dashed line indicates omitted steps. The CimA pathway is highlighted in blue. CimA encodes citramalate synthase; leuCD encodes 2-isopropylmalate isomerase; leuB encodes 3-isopropylmalate dehydrogenase; asp $C$ encodes aspartate aminotransferase; thrA encodes aspartate kinase/homoserine dehydrogenase; asd encodes aspartate semialdehyde dehydrogenase; $\operatorname{th} r B$ encodes homoserine kinase; $\operatorname{thr} C$ encodes threonine synthase; ilvA encodes threonine deaminase; $t d c B$ encodes threonine dehydratase; ilvGM encodes acetohydroxybutanoate synthase; $i l v C$ encodes acetohydroxy acid isomeroreductase; $i l v D$ encodes dihydroxy acid dehydratase; kivd encodes ketoisovalerate decarboxylase; adh2 encodes alcohol dehydrogenase; ilvB/ilvI encodes the subunit of acetolactate synthase; metA encodes homoserine O-succinyltransferase; $t d h$ encodes threonine dehydrogenase

tion [3]. With the identification of these limiting steps, systematic approaches were taken to further improve the $n$-butanol and $n$-propanol co-production in $E$. coli through deregulation of amino acid biosynthesis and elimination of competing pathways [23]. More specifically, the operon consisting of feedback-resistant thrA and thrBC was overexpressed to relieve the threonine feedback inhibition; the host native genes $m e t A$ and $t d h$ were disrupted to prevent the carbon flux leakage out of threonine biosynthetic 
pathway. The genes $i l v B$ and $i l v I$ were also knocked out to avoid the divergence of ketobutyrate to isoleucine, valine, and leucine biosynthesis. With these efforts, a production titer of $2 \mathrm{~g} / \mathrm{l}$ with a nearly $1: 1$ ratio of $n$-butanol and $n$-propanol was achieved.

In addition to the threonine pathway, an alternative pathway was identified in Leptospira interrogans and Methanocaldococcus jannaschii contributing the ketobutyrate formation $[8,13]$. The enzyme citramalate synthase $(\operatorname{Cim} A)$ plays a key role in this pathway (Fig. 5), which directly converts pyruvate to ketobutyrate. This pathway represents a shorter keto acid-mediated pathway to produce $n$-propanol and $n$-butanol from glucose. Atsumi and Liao [4] took advantage of the growth phenotype associated with keto acid deficiency and developed a growth-based screening method to evolve CimA from Methanocaldococcus jannaschii. The best $\operatorname{Cim} A$ mutant demonstrated both insensitivity to isoleucine feedback inhibition and higher catalytic activity, which enabled 22- and 9-fold increases in $n$-butanol and $n$-propanol production compared to wild type $\operatorname{CimA}$. The highest titer of $n$-propanol and $n$-butanol reported in this work were around 3.5 and $0.5 \mathrm{~g} / \mathrm{l}$, respectively.

\section{$2 \mathrm{MB}$ and $3 \mathrm{MB}$ production in $E$. coli}

The keto aid pathways also enable the biosynthesis of 5 -carbon alcohols, including $2 \mathrm{MB}$ and $3 \mathrm{MB}$. Their gramlevel production in recombinant $E$. coli was reported recently [7, 10]. Production of $2 \mathrm{MB}$ in E. coli harnesses isoleucine biosynthesis. It shares the common intermediate, ketobutyrate, with $n$-propanol and $n$-butanol production. Furthermore, 2MB production also shares the ilvIHCD pathway with isobutanol production (Fig. 5). Therefore, to achieve its hyper and selective production is challenging. To shift the carbon flux towards $2 \mathrm{MB}$ biosynthesis, the approaches proven to be effective for improving ketobutyrate availability in the $n$-propanol and $n$-butanol work were still applicable, which included overexpression of thrABC operon and deletion of metA and $t d h$. Furthermore, leu$A B C D$ operon was disrupted to improve the product specificity. Exploring the biodiversity of enzymes catalyzing the key reactions from threonine to KMV, the authors found that overexpression of Salmonella typhimurium AHAS II (ilvGM) and Corynebacterium glutamicum threonine deaminase $(i l v A)$ was more suitable for $2 \mathrm{MB}$-specific production. Combining these approaches, the engineered strain produced $1.25 \mathrm{~g} / 12 \mathrm{MB}$ in $24 \mathrm{~h}[7]$.

Similarly, leucine biosynthetic pathway leading to $3 \mathrm{MB}$ production is an extension of the valine pathway that was engineered for isobutanol production (Fig. 3). 3MB and isobutanol compete for the same substrate KIV. Improving 3MB-specific production in E. coli requiring redistribution of carbon flux between the two branches remained chal- lenging, since the isobutanol production was so efficient already. Overexpressing $E$. coli native $l e u A B C D$ operon did not cause much improvement of $3 \mathrm{MB}$-specific production. This was mainly due to the feedback inhibition of free leucine on the activity of 2-isopropylmalate synthase. Thus, leucine-resistant leuA mutant was employed. In addition, the genes $\operatorname{ty} B$ and $i l v E$ were further disrupted in the previously created isobutanol high producer JCL260 to prevent valine and leucine formation. When the JCL260 $\Delta i l v E \Delta$ tyr $B$ strain expressing the mutated $l e u A$ along with als $S$ $i l v C D$ was examined for $3 \mathrm{MB}$ production, a final titer of $1.28 \mathrm{~g} / \mathrm{l} 3 \mathrm{MB}$ was obtained in $28 \mathrm{~h} \mathrm{[10]}$.

\section{The fatty acid biosynthesis pathway}

Fatty acid biosynthesis is a complicated machinary [18] using acetyl-CoA as a starting molecule. Acetyl-coA is converted to malonyl-CoA by the addition of a carboxyl group using acetyl-CoA carboxylase as a catalyst. The acetyl and malonyl groups on acetyl-CoA and malonyl$\mathrm{CoA}$ are transferred to a small protein called acyl carrier protein (ACP), which has 77 amino acid residues with a phosphopantetheine group specificially attached to a serine residue. Acetyl-ACP (or acetyl-CoA) and Malonyl-ACP are condensed (mediated by $\beta$-ketoacyl-ACP synthases) to generate acetoacyl-ACP. This molecule then goes through reduction, dehydration, and another reduction step to form a 2,3,4-saturated fatty acyl-ACP. In each round of elongation, malonyl-ACP is added to the fatty acyl-ACP to add two carbons. The branch from the saturated to unsaturated pathway occurs when the chain length is ten carbon atoms. The fatty acids synthesized have a long carbon chain backbone, which stores a large amount of energy. In order to transform fatty acids into combustible fuels, pathways leading to biodiesels and long-chain alkanes/alkenes were proposed (Fig. 6). The esterification of fatty acyl-CoA with short chain alcohols generated biodiesels, which can be catalyzed by non-specific acyltransferases. On the other hand, the fatty acyl-CoA can be reduced to the corresponding fatty aldehydes [24], which are in turn decarbonylated to long-chain alkanes or further reduced to fatty alcohols that can also be esterified to biodiesel with acetyl-CoA by an alcohol acyltransferase or ester synthase $[1,20]$.

Fatty acid derivatives: biodiesels and long-chain alkanes/alkenes

As a possible substitute for petroleum-based diesel fuel, biodiesel is made from plant oils through transesterification of triacylglycerols with methanol or ethanol. The generated products are also called fatty acid methyl esters or fatty acid ethyl esters (FAEEs). Although claiming attractive 
Fig. 6 Schematic illustration of fatty acid pathway for biodiesel and long-chain alkanes/alkenes production. The dashed line indicates omitted steps. acp, acyl carrier protein; $a c c A B C D$, acetyl-CoA carboxylase; $f a b D$, malonyl-CoA-ACP transacylase; $f a b H$, ketoacyl-ACP synthase III; $f a b B$, ketoacyl-ACP synthase I; $f a b F$, ketoacyl-ACP synthase II; $f a b G$, ketoacyl-acp reductase; fabA, hydroxydecanoyl-ACP dehydrase; fabZ, hydroxyacyl-ACP dehydratase; $f a b I$, enoyl-ACP reductase

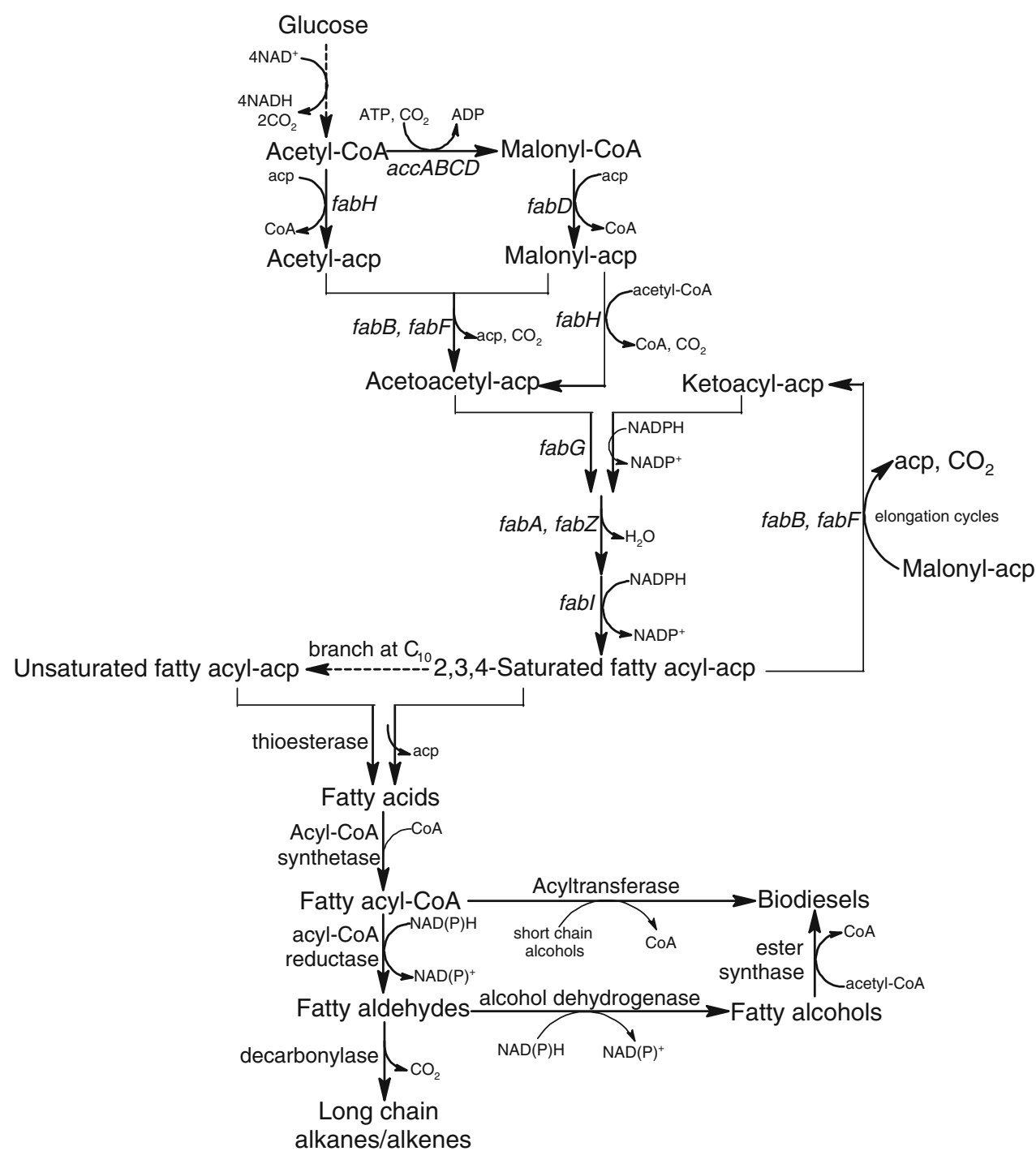

petroleum-diesel-like properties and positive ecological effects, large-scale application of biodiesel seems difficult because of the need to circumvent the geographical and seasonal restrictions, as well as the current costliness of the transesterification procedure [17]. To overcome these drawbacks, Kalscheuer et al., engineered E. coli to produce FAEEs. The traditional ethanol pathway consisting of pyruvate decarboxylase and alcohol dehydrogenase was introduced into $E$. coli to supply ethanol as building units. Subsequent esterification of ethanol with the acyl moieties of coenzyme-A thioesters of fatty acids was achieved by co-expressing a non-specific acyltransferase from Acinetobacter baylyi ADP1. The metabolically engineered E. coli strain was reported to be able to produce FAEEs at a titer of $1.28 \mathrm{~g} / \mathrm{l}$, accounting for $26 \%$ of cellular dry mass by using glucose and oleic acid as substrates.

Recently, production of fatty acid derivatives as biofuels has also been reported in patent applications (e.g., Keasling et al., patent application, WO/2007/136762); this process harnessed the existing fatty acid synthesis machinery in $E$. coli. The E. coli LS9001 was first engineered from membrane-protein-friendly host C41(DE3) by disrupting the $f a d E$ gene. The resulting strain was not capable of degrading fatty acids and fatty acyl-CoAs. As control, the constructed host was able to produce fatty alcohol at amounts of only $0.2-0.5 \mathrm{mg} / \mathrm{l}$. When the acyl-CoA reductase gene acrI from A. baylyi ADP1 and acyl-CoA synthetase gene $f a d D$ from $E$. coli were overexpressed, a fivefold increase in fatty alcohol production was observed. An additional increase was achieved by co-expressing $E$. coli acc $A B C D$ and tes $A$, which encode acetyl-CoA carboxylase and thioesterase. To produce wax ester, the wax synthase gene from A. baylyi ADP1 was introduced into constructed fatty alcohol production strain for overexpression. The intracellular wax yield of $10 \mathrm{mg} / \mathrm{l}$ was reported with 50-ml shake flask fermentation. More interestingly, selection of various thioesterases and modulation of fatty acid biosynthesis allow for the production of fatty acid derivatives with defined 
carbon chain length, saturation points, and branch points. Similarly, in the patent application WO/2008/113041, Friedman and Rude identified four genes (oleA, oleB, oleC, and oleD) from Stenotrophomonas maltophilia that encode proteins involved in the biosynthesis of hydrocarbons, such as olefins and hydrocarbon intermediates, such as aliphatic ketones, by shunting fatty acid biosynthesis. Overexpression of oleA, oleC, and oleD in E. coli $\mathrm{C} 41(\mathrm{DE} 3)$ resulted in olefin production at $7.5 \mathrm{mg} / \mathrm{l}$ with carbon chain length ranging from 27 to 31 . The highest titer of $32 \mathrm{mg} / \mathrm{l}$ olefins was achieved when the host strain was modified by overexpressing $f a d D$ and tesA, and deleting $f a d E$. The patent also disclosed the approaches of cracking olefins and fatty acid esters to short-chain hydrocarbons as fuels and specialty chemicals.

\section{Isoprenoid pathway}

Isoprenoids are natural hydrocarbons biosynthesized for a wide variety of functions, ranging from electron transport cofactors to light harvesting antenna in photosynthetic organisms. The pathway has been well characterized [22] and engineered in heterologous hosts to produce nutraceuticals, such as lycopene, [11] or pharmaceuticals, such as artemisinine [21]. Despite their diversity, isoprenoids are synthesized from two common precursors, isoprenyl diphosphate (IPP) and dimethylallyl pyrophosphate (DMAPP). The IPP and DMAPP are either synthesized from glyceraldehydes-3-phosphate and pyruvate via the methylerythritol pathway or from acetyl-CoA via the mevalonate pathway (Fig. 7) [5].

Isoprenoid derivatives as biofuels

Recently, Withers et al. reported two genes in B. subtilis 6051 whose products can convert the prenyl diphosphate precursors to corresponding isoprentenols [25]. On this basis, the patent application US2008/0092829 has been filed by Renninger et al. for the production of unsaturated C5 alcohols, mainly, 3-methyl-3-buten-1-ol, and 3-methyl2-buten-1-ol. The patent application also disclosed over ten schemes to produce corresponding derivatives using these two alcohols via chemical reactions. The reported titer in the open literature for isoprentenol is relatively low, only $110 \mathrm{mg} / \mathrm{l}$ with a production rate of $2.6 \mathrm{mg} / \mathrm{l} / \mathrm{h}$ [25]. With industrial efforts, the titer reached $1.2 \mathrm{~g} / \mathrm{l}$.

\section{Conclusion}

Although native organisms may produce a desired compound, they typically regulate the production strictly for

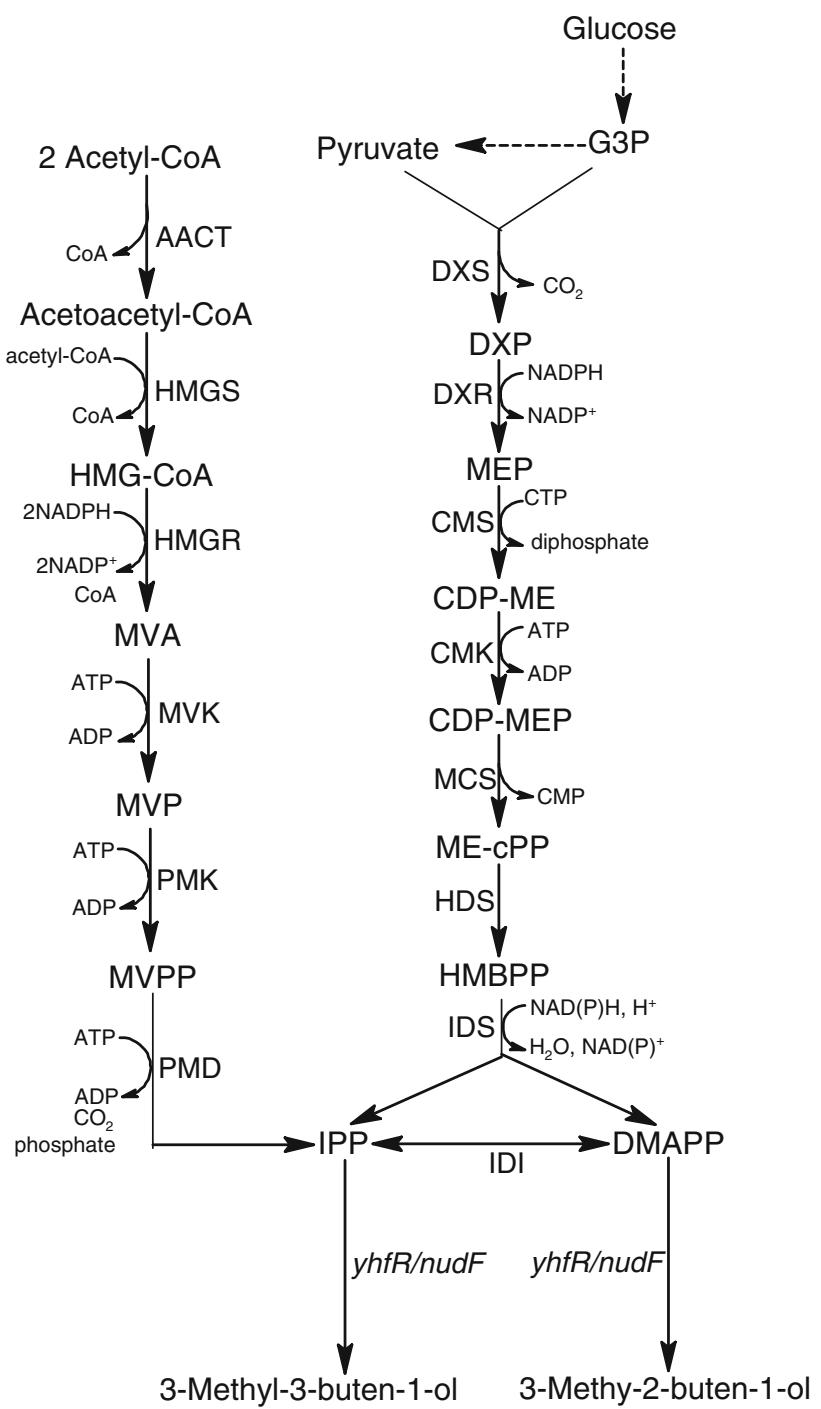

Fig. 7 Schematic illustration of isoprenoid biosynthesis including the mevalonate pathway and methylerythritol pathway. The dashed line indicates omitted steps. $y h f R$ or $n u d F$ encoding prenyl phosphatase. $G 3 P$ glyceraldehyde-3-phosphate, DXP 1-deoxyxylulose-5-phosphate, $M E P$ methylerythritol-4-phosphate, $C D P-M E$ diphosphocytidyl-2methylerythritol, $C D P-M E P$ diphosphocytidyl-2-methylerythritol-2phosphate, $M E-c P P$ methylerythritol cyclopyrophosphate, $H M B P P$ 1-hydroxy-2-methyl-2-(E)-butenyl-pyrophosphate, $H M G$-CoA 3-hydroxy-3-methylglutaryl-CoA, $M V A$ mevalonate, $M V P$ mevalonate-5phosphate, MVPP mevalonate-5-diphosphate, IPP isopentenylpyrophosphate, DMAPP dimethylallyl diphosphate, DXS DXP synthase, DXR DXP reductoisomerase, CMS methylerythritol-4-phosphate cytidyltransferase, $C M K$ 4-diphosphocytidyl-2-methylerythritol kinase, MCS methylerythritol cyclodiphosphate synthase, HDS 1-hydroxy-2-methyl-2-(E)-butenyl-4-diphosphate synthase, IDS IPP/ DMAPP synthase, AACT acetoacetyl-CoA thiolase, HMGS HMG-CoA synthase, $H M G R$ HMG-CoA reductase, $M V K$ MVA kinase, $P M K$ MVP kinase, $P M D$ MVPP decarboxylase, IDI IPP/DMAPP isomerase

their benefit of growth and survival. Therefore, manipulating a native producer for the production purpose may face challenges that limit process improvement. Large-scale transfer and modification of metabolic pathways have 
become a powerful approach for production of metabolites from renewable sources. As such, metabolic engineers (or synthetic biologists) can readily design metabolic systems that combine genes from several different organisms for the production of fuels in user-friendly organisms. Such practice can both serve as a proof of concept and generate a production host in a short time scale.

The metabolic systems discussed above represent the four routes for production of advanced biofuels. On the basis of published literature to date, the keto acid pathway appears to be the most promising, since the isobutanol production has reached a titer of more than $20 \mathrm{~g} / 1$ [3]. This pathway branches out from amino acid biosynthesis, which carries significance. The large-scale production of amino acids has enjoyed many decades of commercial success, indicating that the flux through these pathways can be readily manipulated. Because production of isobutanol mainly occurs in the stationary phase, the flux diversion has minimal impact on growth. Since amino acid biosynthesis exists in almost all microorganisms, the keto acid pathway can be implemented in many different organisms for utilizing different raw materials. The keto acid platform technology opens the possibility for producing many higher alcohols, which can be readily dehydrated to yield hydrocarbons. Thus, this platform technology can be used for making biogasoline, bio-jet fuel, and biodiesel, as well as chemical feed stocks.

Open Access This article is distributed under the terms of the Creative Commons Attribution Noncommercial License which permits any noncommercial use, distribution, and reproduction in any medium, provided the original author(s) and source are credited.

\section{References}

1. Aarts MG, Keijzer CJ, Stiekema WJ, Pereira A (1995) Molecular characterization of the CERl gene of Arabidopsis involved in epicuticular wax biosynthesis and pollen fertility. Plant Cell 7:2115-2127

2. Atsumi S, Cann AF, Connor MR, Shen CR, Smith KM, Brynildsen MP, Chou KJ, Hanai T, Liao JC (2008) Metabolic engineering of Escherichia coli for 1-butanol production. Metab Eng 10:305311. doi:10.1016/j.ymben.2007.08.003

3. Atsumi S, Hanai T, Liao JC (2008) Non-fermentative pathways for synthesis of branched-chain higher alcohols as biofuels. Nature 451:86-89. doi:10.1038/nature06450

4. Atsumi S, Liao JC (2008) Directed evolution of Methanococcus jannaschii citramalate synthase for biosynthesis of 1-propanol and 1-butanol by Escherichia coli. Appl Environ Microbiol 74:78027808. doi:10.1128/AEM.02046-08

5. Atsumi S, Liao JC (2008) Metabolic engineering for advanced biofuels production from Escherichia coli. Curr Opin Biotechnol 19:414-419. doi:10.1016/j.copbio.2008.08.008

6. Bermejo LL, Welker NE, Papoutsakis ET (1998) Expression of Clostridium acetobutylicum ATCC 824 genes in Escherichia coli for acetone production and acetate detoxification. Appl Environ Microbiol 64:1079-1085
7. Cann AF, Liao JC (2008) Production of 2-methyl-1-butanol in engineered Escherichia coli. Appl Microbiol Biotechnol 81:8998. doi:10.1007/s00253-008-1631-y

8. Charon NW, Johnson RC, Peterson D (1974) Amino acid biosynthesis in the Spirochete Leptospira: evidence for a novel pathway of isoleucine biosynthesis. J Bacteriol 117:203-211

9. Chen J-S, Hiu SF (1986) Acetone-butanol-isopropanol by Clostridium beijerinckii (synonym, Clostridium butylicum). Biotechnol Lett 8:371-376. doi:10.1007/BF01040869

10. Connor MR, Liao JC (2008) Engineering of an Escherichia coli strain for the production of 3-methyl-1-butanol. Appl Environ Microbiol 74:5769-5775. doi:10.1128/AEM.00468-08

11. Farmer WR, Liao JC (2000) Improving lycopene production in Escherichia coli by engineering metabolic control. Nat Biotechnol 18:533-537. doi:10.1038/75398

12. Hanai T, Atsumi S, Liao JC (2007) Engineered synthetic pathway for isopropanol production in Escherichia coli. Appl Environ Microbiol 73:7814-7818. doi:10.1128/AEM.01140-07

13. Howell DM, Xu H, White RH (1999) (R)-citramalate synthase in methanogenic archaea. J Bacteriol 181:331-333

14. Ingram LO, Conway T, Clark DP, Sewell GW, Preston JF (1987) Genetic engineering of ethanol production in Escherichia coli. Appl Environ Microbiol 53:2420-2425

15. Ingram LO, Gomez PF, Lai X, Moniruzzaman M, Wood BE, Yomano LP, York SW (1998) Metabolic engineering of bacteria for ethanol production. Biotechnol Bioeng 58:204-214. doi:10.1002/(SICI)1097-0290(19980420)58:2/3<204::AID-BIT13 $>3.0 . \mathrm{CO} ; 2-\mathrm{C}$

16. Jarboe LR, Grabar TB, Yomano LP, Shanmugan KT, Ingram LO (2007) Development of ethanologenic bacteria. Adv Biochem Eng Biotechnol 108:237-261

17. Kalscheuer R, Stolting T, Steinbuchel A (2006) Microdiesel: Escherichia coli engineered for fuel production. Microbiology 152:2529-2536. doi:10.1099/mic.0.29028-0

18. Magnuson K, Jackowski S, Rock CO, Cronan JE Jr (1993) Regulation of fatty acid biosynthesis in Escherichia coli. Microbiol Rev 57:522-542

19. Peterson JD, Ingram LO (2008) Anaerobic respiration in engineered Escherichia coli with an internal electron acceptor to produce fuel ethanol. Ann NY Acad Sci 1125:363-372. doi:10.1196/annals.1419.020

20. Riendeau D, Meighen E (1985) Enzymatic reduction of fatty acids and acyl-CoAs to long chain aldehydes and alcohols. Experientia 41:707-713. doi:10.1007/BF02012564

21. Ro DK, Paradise EM, Ouellet M, Fisher KJ, Newman KL, Ndungu JM, Ho KA, Eachus RA, Ham TS, Kirby J, Chang MC, Withers ST, Shiba Y, Sarpong R, Keasling JD (2006) Production of the antimalarial drug precursor artemisinic acid in engineered yeast. Nature 440:940-943. doi:10.1038/nature04640

22. Sacchettini JC, Poulter CD (1997) Creating isoprenoid diversity. Science 277:1788-1789. doi:10.1126/science.277.5333.1788

23. Shen CR, Liao JC (2008) Metabolic engineering of Escherichia coli for 1-butanol and 1-propanol production via the keto-acid pathways. Metab Eng 10:312-320. doi:10.1016/j.ymben.2008. 08.001

24. Wang X, Kolattukudy PE (1995) Solubilization and purification of aldehyde-generating fatty acyl-CoA reductase from green alga Botryococcus braunii. FEBS Lett 370:15-18. doi:10.1016/00145793(95)00781-4

25. Withers ST, Gottlieb SS, Lieu B, Newman JD, Keasling JD (2007) Identification of isopentenol biosynthetic genes from Bacillus subtilis by a screening method based on isoprenoid precursor toxicity. Appl Environ Microbiol 73:6277-6283. doi:10.1128/AEM. 00861-07 